\title{
Correction to: The National Registry of Retinoblastoma in Japan (1983-2014)
}

\author{
The Committee for the National Registry of Retinoblastoma ${ }^{1}$
}

Published online: 22 June 2018

(c) Japanese Ophthalmological Society 2018

\section{Correction to: Japanese Journal of Ophthalmology https://doi.org/10.1007/s10384-018-0597-2}

In the original publication, the address of "The Committee for the National Registry of Retinoblastoma" has been given incorrectly. The correct address is given in this Correction:

The Committee for the National Registry of Retinoblastoma,

Japanese Ophthalmological Society, 2-4-11-402

Kanda-Sarugakucho, Chiyoda-ku, Tokyo 101-8346, Japan

The original article can be found online at https://doi.org/10.1007/ s10384-018-0597-2.

The Committee for the National Registry of Retinoblastoma azuma-n@ncchd.go.jp

1 The Committee for the National Registry of Retinoblastoma, Japanese Ophthalmological Society, 2-4-11-402

Kanda-Sarugakucho, Chiyoda-ku, Tokyo 101-834, Japan 